The discrepancy between (6) and (3) which arises, if the term $\mathrm{d} p / \mathrm{d} s$ in (2) is not neglected, is easily remedied by allowing for the creation of matter by the action of pressure in the Lagrange equation of continuity (5) also, by writing it :

$$
\rho \Gamma \mathrm{d}^{3} u-\rho_{V\left(\Gamma \mathrm{d}^{3} u\right)}^{-\rho_{1} \Gamma_{1} \mathrm{~d}^{3} u+} \frac{\mathrm{d} p}{\mathrm{~d} s} \mathrm{~d} \tau=0 .
$$

The volume integration is over the four-dimensional volume swept out by the area $\Gamma d^{3} u$.

A detailed account of the above results together with a discussion of the relationship between the Minkowski tensor and canonical tensor deduced from the Lagrangian used in (4) will be published elsewhere. I thank Prof. P. A. M. Dirac for his interest in this work.

$$
\text { S. F. B. TYABJI }
$$

Christ's College, Cambridge. June 10.

1 Finstein, A., "The Meaning of Relativity" (London, 1950)

"Eddington, A. S., "The Mathematical Theory of Relativity" (Cambridge, 1923).

\section{A Fundamental Law of Geochemistry}

The late V. M. Goldschmidt defined one principal task of geochemistry as being, "to find the laws of distribution of the elements". One such law may apparently be found by close scrutiny of fully quantitative determinations of many elements in a comparatively large number of specimens of some specific rock or mineral type.

The determinations of potas. sium, rubidium, scandium, vanadium, cobalt, gallium, chromium and zirconium in diabase (Fairbairn, Ahrens and Gorfinkle $\left.{ }^{1}\right)$ and potassium, rubidium, cæsium, lead, scandium, vanadium, cobalt, chromium and zirconium (Ahrens ${ }^{2}$ ), molybdenum (Sandell ${ }^{3}$ ) and fluorine (Seraphim ${ }^{4}$ ) in granite have been examined statistically, and the conclusions reached are: first, frequency distribution plots of the usual type (for example, Fig. 1) assume a decided positive skewness when the dispersion of an element is large--hence, distribution is not normal although it may appear to be so for those elements which show small dispersion (for example, Fig. 2) ; secondly, distribution becomes normal in all examples, irrespective of the magnitude of the dispersion, when concentration (the variate) is transformed to log concentration (see Figs. 1 and 2). As the total number of observations is reasonably large (812) and as they refer to a variety of distinctly different elements, the following fundamental geochemical law concerning the nature of the distribution of elements may be enunciated with some confidence:
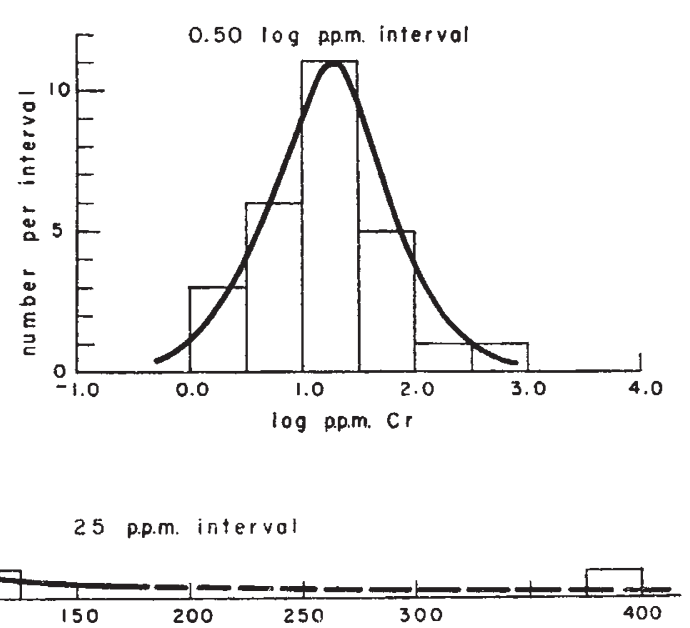
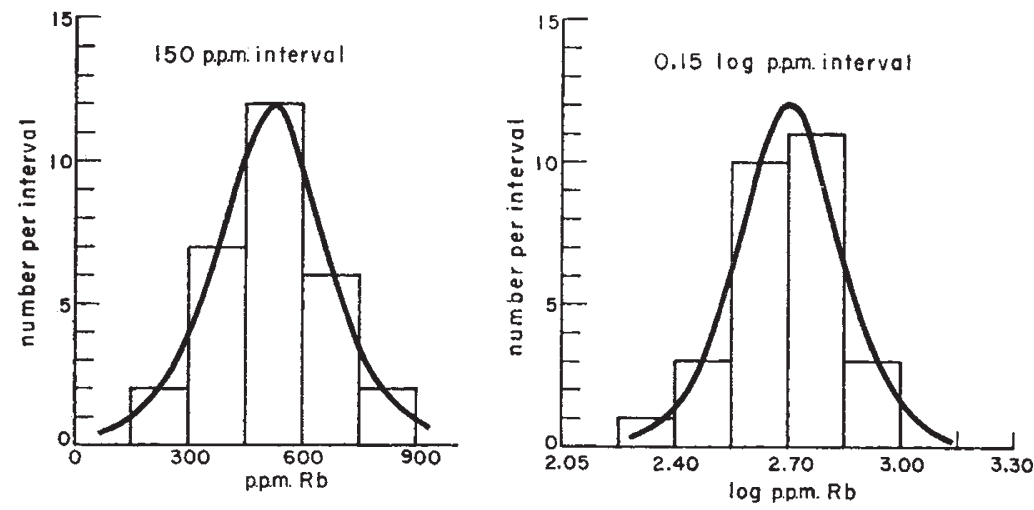

the concentration of an element is lognormally distributed in a specific igneous rock.

It follows directly from this law that the abundance (arithmetic mean or average) of an element is always greater than the most prevalent concentration, which in a lognormal distribution is given by the geometric mean. The difference is determined solely by the magnitude of the dispersion. In the above examples, the abundance and most prevalent concentration of rubidium are virtually identical, whereas the abundance of chromium (40 p.p.m.) is more than twice its most prevalent concentration (17 p.p.m.).

Fig. 2. Rubidium in New England granite. Both the rubidium content and its logarithm appear normally distributed. Dispersion is small $(C=28$ per cent $)$

A full discussion will be published elsewhere.

L. H. Amrens

Department of Geology and Geophysics, Massachusetts Institute of Technology, Cambridge 39, Mass. Oct. 9 .

${ }^{2}$ Fairbairn, H. W.. Ahrens, L. H., and Gorfinkle, L. G., Geachim. Cosmochim. Acta, 3, 34 (1953).

- Ahrens, L. H. (in preparation for publication).

- Sandell, E. B., and Kuroda, P. K., Office of Naval Research (Washington) Report, NR081-132 (1952).

' Seraphim, R. H., Ph.D. thesis, Dept. of Geology and Geophysics M.I.T. (1951). 